\title{
Quasitriangularity of Quantum Groups at Roots of 1
}

\author{
N. Reshetikhin ${ }^{1}$ \\ Department of Mathematics, University of California, Berkeley, CA 94720, USA
}

Received: 29 March 1994/in revised form: 26 August 1994

\begin{abstract}
An important property of a Hopf algebra is its quasitriangularity and it is useful for various applications. This property is investigated for quantum groups $s l_{2}$ at roots of 1 . It is shown that different forms of the quantum group $s l_{2}$ at roots of 1 are either quasitriangular or have similar structure which will be called braiding. In the most interesting cases this property means that "braiding automorphism" is a combination of some Poisson transformation and an adjoint transformation with a certain element of the tensor square of the algebra.
\end{abstract}

Algebras which here will be called quantum $s l_{2}$ are the simplest examples of quantum groups which have practically all the remarkable properties of this class of Hopf algebras. One of the most important properties of quantum groups is quasitriangularity. Recall the definition from [Dr].

Definition 1. A Hopf algebra $A$ is called quasitriangular if there exists $R \in$ $A \otimes A$ (or an element from the appropriate completion of $A \otimes A)$ such that

$$
\begin{aligned}
\Delta^{\prime}(a) & =R \Delta(a) R^{-1}, \\
(\Delta \otimes \mathrm{id})(R) & =R_{13} R_{23}, \\
(\mathrm{id} \otimes \Delta)(R) & =R_{13} R_{12} .
\end{aligned}
$$

Here $\Delta^{\prime}(a)=\sigma \circ \Delta(a)$, where $\sigma: A^{\otimes 2} \rightarrow A^{\otimes 2}, a \otimes b \mapsto b \otimes a$ and $R_{12}, R_{13}, R_{23} \in$ $A^{\otimes 3}$ (or to the appropriate completion of it), $R_{12}=R \otimes 1, R_{23}=1 \otimes R, R_{13}=$ $(\sigma \otimes \mathrm{id})\left(R_{23}\right)=(\mathrm{id} \otimes \sigma)\left(R_{12}\right)$.

A remarkable corollary of this definition is that $R$ satisfies the Yang-Baxter equation in $A^{\otimes 3}$ :

$$
R_{12} R_{13} R_{23}=R_{23} R_{13} R_{12}
$$

It is known [Dr] that quantum universal enveloping algebras $U_{h} \mathfrak{g}$ are quasitriangular over $\mathbb{C}[[h]]$ for any Kac-Moody algebra $\mathfrak{g}$. It is also known that the

1 This work was supported by an Alfred P. Sloan fellowship and by National Science Foundation grant DMS-9296120. 
corresponding algebraic quantum universal algebra $U_{q}(\mathfrak{g})$ introduced by Jimbo $[\mathrm{J}]$ for generic $q$ are not quasitriangular if considered over $\mathbb{C}\left[q, q^{-1}\right]$. This fact is essential when $q$ is specialized at roots of 1 .

In this paper the quasitriangularity of Hopf algebras $U_{q}(\mathfrak{g})$ will be studied for $\mathfrak{g}=s l_{2}$. It is studied over $\mathbb{C}\left[q, q^{-1}\right]$ for general values of $q$ and when $q$ is a root of 1 .

The main result is that for algebras $U_{q}(\mathfrak{g})$ we have a somewhat more general property than quasitriangularity (1)-(3).

Definition 2. A Hopf algebra $A$ is called braided if there exists an automorphism $R$ of $A \otimes A$ (or of an appropriate completion of $A \otimes A$ ) distinct from $\sigma: a \otimes b \mapsto$ $b \otimes a$ such that

$$
\begin{aligned}
\Delta^{\prime}(a) & =R(\Delta(a)), \\
(\Delta \otimes \mathrm{id}) \circ R & =R_{13} \circ R_{23} \circ(\Delta \otimes \mathrm{id}), \\
(\mathrm{id} \otimes \Delta) \circ R & =R_{13} \circ R_{12} \circ(\mathrm{id} \otimes \Delta) .
\end{aligned}
$$

Here $R_{12}, R_{13}, R_{23}$ are automorphisms of $A \otimes A \otimes A$ such that $R_{12}=R \otimes \mathrm{id}, R_{23}=$ id $\otimes R, R_{13}=(\sigma \otimes \mathrm{id}) \circ(\mathrm{id} \otimes R) \circ(\sigma \otimes \mathrm{id})$.

It follows from this definition that the automorphism $R$ satisfies the Yang-Baxter equation in $\operatorname{End}\left(A^{\otimes 3}\right)$ :

$$
R_{12} \circ R_{13} \circ R_{23}=R_{23} \circ R_{13} \circ R_{12} \text {. }
$$

Let $R^{(0)}$ above be an exterior automorphism of $A \otimes A$ and $R^{(1)} \in A \otimes A$ be an invertible element. Consider the automorphism

$$
a \mapsto R^{(1)} a R^{(1)^{-1}}, \quad a, R^{(1)} \in A \otimes A .
$$

Definition 3. The element $R^{(1)}$ is a universal $R$-matrix of the braided Hopf algebra $\left(A, R^{(0)}, R^{(1)}\right)$ if

$$
\Delta^{\prime}(a)=R^{(0)}\left(R^{(1)} \Delta(a) R^{(1)^{-1}}\right) .
$$

(ii) (5) and (6) hold for $R^{(0)}$

$$
\begin{aligned}
& (\Delta \otimes \mathrm{id})\left(R^{(1)}\right)=R_{23}^{(0)^{-1}}\left(R_{13}^{(1)}\right) R_{23}^{(1)}, \\
& (\mathrm{id} \otimes \Delta)\left(R^{(1)}\right)=R_{12}^{(0)^{-1}}\left(R_{13}^{(1)}\right) R_{12}^{(1)} .
\end{aligned}
$$

Now for the universal $R$-matrix we will have the following relations:

$$
\begin{aligned}
& \left(R_{12}^{(0)^{-1}} \circ R_{13}^{(0)^{-1}}\right)\left(R_{23}^{(1)}\right) \cdot R_{12}^{(0)^{-1}}\left(R_{13}^{(1)}\right) \cdot R_{12}^{(1)}=R_{12}^{(1)} R_{23}^{(0)^{-1}}\left(R_{13}^{(1)}\right) R_{23}^{(1)} \text { ， } \\
& \left(R_{23}^{(0)^{-1}} \circ R_{13}^{(0)^{-1}}\right) R_{12}^{(1)} \cdot R_{23}^{(0)^{-1}}\left(R_{13}^{(1)}\right) \cdot R_{23}^{(1)}=R_{23}^{(1)} \cdot R_{12}^{(0)^{-1}}\left(R_{13}^{(1)}\right) \cdot R_{12}^{(1)} \text {. }
\end{aligned}
$$

We will say that the decomposition

$$
R(a)=R^{(0)}\left(R^{(1)} a R^{(1)^{-1}}\right)
$$

is a regular splitting of $R$ if

$$
\begin{aligned}
& \left(R_{23}^{(0)^{-1}} \circ R_{13}^{(0)^{-1}}\right)\left(R_{12}^{(1)}\right)=R_{12}^{(1)}, \\
& \left(R_{12}^{(0)^{-1}} \circ R_{13}^{(0)^{-1}}\right)\left(R_{23}^{(1)}\right)=R_{23}^{(1)} .
\end{aligned}
$$


If $R^{(0)}$ and $R^{(1)}$ is a regular splitting of $R$ we have the following relation for $R^{(1)}$ :

$$
R_{12}^{(1)} R_{23}^{(0)^{-1}}\left(R_{23}^{(1)}\right) R_{23}^{(1)}=R_{23}^{(1)} R_{12}^{(0)}\left(R_{13}^{(1)}\right) R_{12}^{(1)} .
$$

In the first section we recall the definition and the main properties of the formal deformation $U_{h} s l_{2}$ of $U\left(s l_{2}\right)$. This section also contains the description of the "algebraic" quantum group $U_{q}\left(s l_{2}\right)$ and its main properties including braiding. In the second section the algebra $U_{q}\left(s l_{2}\right)$ and its relation to $U\left(s l_{2}\right)$ are studied further. The third section contains facts about quantum $s l_{2}$ at roots of 1 and the description of the braiding of this quantum group.

The $s l_{2}$ case is chosen for simplicity. The corresponding properties of $U_{q}(\mathfrak{g})$ for simple Lie algebras $\mathfrak{g}$ as well as an explicit description of the center for $U_{q}(\mathfrak{g})$ when $q$ is a root of 1 will be done in a forthcoming publication.

This work was finished during a visit by the author to the School of Mathematics and School of Theoretical Physics at the Australian National University at Canberra. I would like to thank R. Baxter and V. Bazhanov for their hospitality. It is also my pleasure to thank R. Baxter, V. Bazhanov, J. Mattes and I. Frenkel for valuable discussions and remarks.

\section{Quantum $s l_{2}$ over $\mathbb{C} \mid\left[h \|\right.$ and $\mathbb{C}\left[q, q^{-1}\right]$}

1.1. Here we recall the definition and the main properties of the algebra which is called quantum $s l_{2}$ and is denoted as $U_{h} s l_{2}$. This is a $\mathbb{C}[[h]]$-algebra generated by elements $H, X, Y$ with the following determining relations:

$$
\begin{gathered}
{[H, X]=2 X, \quad[H, Y]=-2 Y,} \\
{[X, Y]=\frac{\operatorname{sh}\left(\frac{h H}{2}\right)}{\operatorname{sh}\left(\frac{h}{2}\right)} .}
\end{gathered}
$$

This algebra is a Hopf algebra with the comultiplication

$$
\begin{aligned}
& \Delta H=H \otimes 1+1 \otimes H, \\
& \Delta X=X \otimes e^{h H / 4}+e^{-h H / 4} \otimes X, \\
& \Delta Y=Y \otimes e^{h H / 4}+e^{-h H / 4} \otimes Y .
\end{aligned}
$$

The algebra $U_{h} s l_{2}$ is a formal deformation of $U s l_{2}$ :

$$
U_{h} s l_{2} / h U_{h} s l_{2} \simeq U s l_{2} .
$$

The algebra $U_{h} s l_{2}$ is quasitriangular with $R \in U_{h} s l_{2} \hat{\otimes} U_{h} s l_{2}$ (where $\hat{\otimes}$ is the $h$-adic completion of $U_{h} s l_{2}^{\otimes 2}$ ) given as follows:

$$
\begin{aligned}
R= & \exp \left(\frac{H \otimes H}{4}\right) \sum_{k \geqq 0} \frac{\left(e^{h}-1\right)^{2 k}}{\left(e^{h k}-1\right)\left(e^{h(k-1)}-1\right) \ldots\left(e^{h}-1\right)} e^{\frac{h}{2} n(n-3)} \\
& \times\left(e^{\frac{h H}{4}} X\right)^{k} \otimes\left(e^{-\frac{h H}{4}} Y\right)^{k} .
\end{aligned}
$$

The representation theory of the algebra $U_{h} s l_{2}$ is the same as the representation theory of $U_{s} l_{2}[[h]]$ due to the following fact $[\operatorname{Dr} 1, \mathrm{~J}]$. 
Proposition 1.1.2. There is an isomorphism of algebras

$$
U_{h} s l_{2} \stackrel{\sim}{\rightarrow} U_{S} l_{2}[[h]] .
$$

Any map $H \mapsto H, X \mapsto X \psi_{h}(H, c), Y \mapsto Y \phi_{h}(H, c)$, where $\phi_{h}(z, c)=\psi_{h}(z, c)=1$ $\bmod h(\phi, \psi \in \mathbb{C}[z, c][[h]])$ and

$\psi_{h}(z-2, c) \phi_{h}(z)\left(c-H^{2} / 2+H\right)-\phi_{h}(z+2, c) \psi_{h}(z, c)\left(c-H^{2} / 2-H\right)=2 \frac{\operatorname{sh}\left(\frac{h}{2}\right)}{\operatorname{sh}\left(\frac{h z}{2}\right)}$ provides such an isomorphism.

The category $U_{h} s l_{2}$-mod of finite-dimensional $U_{h} s l_{2}$-modules is an abelian category over $\mathbb{C}[[h]]$. Objects of this category are pairs $(V, \pi)$, where $V$ is a finitedimensional $\mathbb{C}[[h]]$-vectorspace and $\pi: U_{h} s l_{2} \rightarrow \operatorname{End}(V)$ is a homomorphism of algebras (abusing notations we will say $V$ is a vectorspace even in the case when it is a module just over a ring, not a field). Morphisms $f:\left(V_{1}, \pi_{1}\right) \rightarrow\left(V_{2}, \pi_{2}\right)$ are $\mathbb{C}[[h]]$-linear maps from $V_{1}$ to $V_{2}$ which are also $U_{h} s l_{2}$-linear: $f \pi_{1}(a)=\pi_{2}(a) f$ for each $a \in U_{h} s l_{2}$.

The category $U_{h} s l_{2}$-mod is a rigid monoidal category with identity object $\mathbb{1} \simeq$ $\mathbb{C}[[h]]$ and with the tensor product $\left(V_{1}, \pi_{1}\right) \otimes\left(V_{2}, \pi_{2}\right)=\left(V_{1} \otimes V_{2},\left(\pi_{1} \otimes \pi_{2}\right) \circ \Delta\right)$. The object dual to $(V, \pi)$ is a pair $\left(V^{*}, \pi^{*} \circ S\right)$, where $V^{*}$ is a vectorspace dual to $V$ over $\mathbb{C}[[h]]$ and $\pi^{*}(a)$ is a dual $\mathbb{C}[[h]]$-linear map to $\pi(a)$. The category $U_{h} s l_{2}$ mod is a strict monoidal category due to coassociativity of the comultiplication.

The quasitriangularity of the algebra $U_{h} s l_{2}$ implies that the category of $U_{h} s l_{2}$ mod is a braided category. The braiding is a collection of functorial isomorphisms

$$
c_{V W}: V \otimes W \rightarrow W \otimes V
$$

where $c_{V W}=P_{V W} \cdot\left(\pi_{V} \otimes \pi_{W}\right)(R)$. Here $P_{V W}(x \otimes y)=y \otimes x$ is a permutation operator, $R$ is the universal $R$-matrix (1.1.7).

The remarkable property of the deformation $U_{h} l_{2}$ of $U s l_{2}$ is that relations between $\exp \left( \pm \frac{h H}{4}\right), X$ and $Y$ are algebraically closed and the action of the comultiplication on them results in an algebraic combination of these elements. Moreover relations between these elements and the comultiplication are defined over $\mathbb{C}\left[e^{ \pm h}\right]$. This observation is a motivation for the algebra described in the next section.

1.2. It turns out that the formal deformation described above also gives a family of Hopf algebras. The algebra $U_{q}\left(s l_{2}\right)$ for an undetermined $q$ as the $\mathbb{C}\left[q, q^{-1}\right]$-algebra is generated by $k, k^{-1}, e$ and $f$ with the following determining relations:

$$
\begin{gathered}
k k^{-1}=k^{-1} k=1, \quad k e=q e k, \\
k f=q^{-1} f k, \quad \text { ef }-f e=\frac{k-k^{-1}}{q-1} .
\end{gathered}
$$

We will call this algebra polynomial quantum $s l_{2}$. This is a Hopf algebra [Dr, J, S1] with the following action of the comultiplication on generators:

$$
\begin{aligned}
\Delta k & =k \otimes k, \\
\Delta e & =e \otimes k+1 \otimes e, \\
\Delta f & =f \otimes 1+k^{-1} \otimes f .
\end{aligned}
$$


Remark. 1.2.1. We have the isomorphism of algebras:

$$
U_{q}\left(s l_{2}\right) \rightleftharpoons U_{q-1}\left(s l_{2}\right)
$$

given by the map

$$
q \mapsto q^{-1}, \quad k \mapsto k^{-1}, \quad e \mapsto e k^{-1}, \quad f \mapsto f k .
$$

Remark. 1.2.2. Since $U_{q}\left(s l_{2}\right)$ is defined over $\mathbb{C}\left[q, q^{-1}\right]$ we can specialize $q$ to any nonzero complex number. Thus $U_{q}\left(s l_{2}\right)$ determines a family of Hopf algebras. Monomials $e^{n} k^{m} f^{l}$ form a linear basis in $U_{q}\left(s l_{2}\right)$. This allows us to identify these algebras as vectorspaces for different complex values of $q$. We assume this identification in the rest of this paper.

1.2.3. Identify $q=e^{h}$ and consider $\mathbb{C}[[h]]$ as a module over $\mathbb{C}\left[q, q^{-1}\right]$. Then we have an injective homomorphism of algebras:

$$
\begin{gathered}
\phi: U_{q}\left(s l_{2}\right) \rightarrow U_{h} s l_{2} \otimes \mathbb{C}[[h]] \\
\left.\phi(k)=e^{\frac{h H}{2}} \otimes \varepsilon, \quad \phi(h]\right][\varepsilon], \quad \varepsilon^{2}=1, \\
\phi(e)=e^{\frac{h H}{4}} X \otimes \varepsilon, \quad \phi(f)=e^{-\frac{h H}{4}} Y \otimes e^{-h} .
\end{gathered}
$$

Here the left side is regarded as the module over $\mathbb{C}[[h]]$.

The following seems well known.

Proposition 1.2.4. The center of $U_{q}\left(s l_{2}\right)$ is generated by the element

$$
c=e f+\frac{k+q k^{-1}}{(q-1)^{2}} \text {. }
$$

The central element (1.2.10) was first constructed in [S2]. The fact that it generates the center of $U_{q}\left(s l_{2}\right)$ can be found in [DK].

1.3. The algebra $U_{q}\left(s l_{2}\right)$ is not quasitriangular in a sense of Definition 1. But, as we will see, it is braided in the sense of Definition 2.

Consider the algebra $U_{q}\left(s l_{2}\right)$ over $\mathbb{C}\left[q^{\frac{1}{2}}, q^{-\frac{1}{2}}\right]$. Define the automorphism $R_{0}$ of $U_{q}\left(s l_{2}\right)^{\otimes 2}$ as follows:

$$
\begin{aligned}
R_{0}(k \otimes 1) & =k \otimes 1, & & R_{0}(1 \otimes k)=1 \otimes k, \\
R_{0}(e \otimes 1) & =e \otimes k, & & R_{0}(1 \otimes e)=k \otimes e, \\
R_{0}(f \otimes 1) & =f \otimes k^{-1}, & & R_{0}(1 \otimes f)=k^{-1} \otimes f,
\end{aligned}
$$

Consider the following completions of $U_{q}\left(s l_{2}\right)$ :

$$
\begin{aligned}
& U_{q}\left(s l_{2}\right)^{(e)}=\left\{\sum_{n=0}^{\infty} e^{n} P_{n}\left(k^{ \pm 1}, f\right)\right\}, \\
& U_{q}\left(s l_{2}\right)^{(f)}=\left\{\sum_{n=0}^{\infty} Q_{n}\left(k^{ \pm 1}, e\right) f^{n}\right\} .
\end{aligned}
$$

Here $P_{n}$ and $Q_{n}$ are polynomials. It is clear that these completions of vectorspaces are indeed completions of algebras. 
Consider the following completion of $U_{q}\left(s l_{2}\right)^{\otimes 2}$ :

$$
U_{q}\left(s l_{2}\right)^{\hat{\otimes} 2}=\left\{\sum_{n, m=0}^{\infty} e^{n} P_{n}\left(k^{ \pm 1}, f\right) \otimes Q_{m}\left(e, k^{ \pm 1}\right) f^{m}\right\} .
$$

Again, this is a completion of the algebra $U_{q}\left(s l_{2}\right)^{\otimes 2}$, such that

Clearly the element

$$
U_{q}\left(s l_{2}\right)^{\hat{\otimes} 2} \hookleftarrow U_{q}\left(s l_{2}\right)^{(e)} \otimes U_{q}\left(s l_{2}\right)^{(f)} .
$$

$$
R_{1}=\sum_{k=0}^{\infty} \frac{(q-1)^{k}}{(k)_{q} !} q^{\frac{k(k-1)}{2}} e^{k} \otimes f^{k},
$$

which is the last factor in the $R$-matrix belongs to $U_{q}\left(s l_{2}\right)^{\hat{\otimes} 2}$. Here $(k)_{q} !=$ $(k)_{q} \ldots(1)_{q},(k)_{q}=\frac{q^{k}-1}{q-1}$. It is also clear that $(1.3 .8)$ is invertible in $U_{q}\left(s l_{2}\right)^{\hat{\otimes} 2}$.

Consider $U_{q}\left(s l_{2}\right)^{\otimes 2}$ as a subspace in $U_{q}\left(s l_{2}\right)^{\hat{\otimes} 2}$.

Proposition 1.3.1. The Hopf algebra $U_{q}\left(s l_{2}\right)$ is braided with

$$
R(a)=R_{0}\left(R_{1} a R_{1}^{-1}\right),
$$

where $R_{0}$ and $R_{1}$ are defined above in (1.3.1)-(1.3.3) and (1.3.8). The element $R_{1}$ is a universal R-matrix for $U_{q}\left(s l_{2}\right)$ (in the sense of Definition 3 ).

Proof. First consider the algebra $U_{h} s l_{2}$ from Sect. 1.1. Denote

$$
\begin{gathered}
R_{0}=\exp \left(\frac{h}{4} H \otimes H\right) \\
R_{1}=\sum_{n \geqq 0} \frac{\left(e^{h}-1\right)^{2 n}}{\left(e^{h n}-1\right) \ldots\left(e^{h}-1\right)} e^{\frac{h}{2} n(n+1)}\left(e^{\frac{h H}{4}} X\right)^{n} \otimes\left(e^{-\frac{h H}{4}} Y\right)^{n} .
\end{gathered}
$$

It is easy to check that

$$
\begin{array}{ll}
R_{0}(X \otimes 1)=\left(X \otimes e^{\frac{h H}{2}}\right) R_{0}, & R_{0}(1 \otimes X)=\left(e^{\frac{h H}{2}} \otimes X\right) R_{0}, \\
R_{0}(Y \otimes 1)=\left(Y \otimes e^{-\frac{h H}{2}}\right) R_{0}, & R_{0}(1 \otimes Y)=\left(e^{-\frac{h H}{2}} \otimes Y\right) R_{0} .
\end{array}
$$

Therefore the automorphism $a \mapsto R_{0} a R_{0}^{-1}$ can be extended from the automorphism of $U_{h} s l_{2}^{\otimes 2}$ to the automorphism of $U_{q}\left(s l_{2}\right)^{\otimes 2}$ (it will be an exterior automorphism of $\left.U_{q}\left(s l_{2}\right)^{\otimes 2}\right)$. Comparing with (1.3.1)-(1.3.3) we identify it with $R_{0}$.

Clearly $R_{0}$ satisfies conditions (5) and (6) and the element $R_{1}$ satisfies (10), (11), (15). Because there is a homomorphism of Hopf algebras $U_{q}\left(s l_{2}\right) \rightarrow U_{h} s l_{2}$, an automorphism (1.3.1)-(1.3.3) is a preimage of $R_{0} a R_{0}^{-1}$ in $U_{h} s l_{2}^{\otimes 2}$ and the element (1.3.8) is a preimage of (1.3.11). We expect Proposition 1.2.3 to be true since the homomorphism is injective.

The other way to prove Proposition 1.2 .3 is by easy direct computation.

It is easy to see that the splitting on $R_{0}$ and $R_{1}$ is a regular splitting in the sense of (15) and therefore $R_{1}$ satisfies the twisted Yang-Baxter equation (16). In Sect. 1.4 this equation will be written explicitly. 
The algebra $U_{q}\left(s l_{2}\right)$ is a deformation of the universal enveloping algebra of $s l_{2}$ in the following sense.

Consider the algebra $U s l_{2}^{(\varepsilon)} \simeq U s l_{2} \otimes_{\mathbb{C}} \mathbb{C}[\varepsilon]$, where $\varepsilon^{2}=1$ and introduce the following Hopf algebra structure on it:

$$
\begin{aligned}
& \tilde{\Delta} H=H \otimes 1+1 \otimes H, \quad \tilde{\Delta}(\varepsilon)=\varepsilon \otimes \varepsilon, \\
& \tilde{\Delta} X=X \otimes 1+\varepsilon \otimes X, \\
& \tilde{\Delta} Y=Y \otimes 1+\varepsilon \otimes Y .
\end{aligned}
$$

Proposition 1.3.2. There is an isomorphism of Hopf algebras:

$$
U_{q}\left(s l_{2}\right) /(q-1) U_{q}\left(s l_{2}\right) \stackrel{\sim}{\rightarrow} U s l_{2}^{(\varepsilon)},
$$

where

$$
\begin{aligned}
\varepsilon & =k \bmod (q-1), & H & =\frac{k^{2}-1}{q-1} \bmod (q-1), \\
X & =k^{-1} e \bmod (q-1), & Y & =f \bmod (q-1) .
\end{aligned}
$$

The proof is clear.

1.4. Consider the function of complex $z$,

$$
(z ; q)_{\infty}=\prod_{n \geqq 1}\left(1-z q^{n}\right) .
$$

We regard it as an element of $\mathbb{C}[[q]]$. This product converges to an analytic function of $z$ in any finite part of $\mathbb{C}$ if $q$ is a complex number, and $|q|<1$.

The following identities are well known:

$$
\begin{aligned}
& (z ; q)_{\infty}=\sum_{n \geqq 0} \frac{(-1)^{n} q^{\frac{n(n+1)}{2}}}{(n)_{q} !(1-q)^{n}} z^{n}, \\
& (z ; q)_{\infty}^{-1}=\sum_{n \geqq 0} \frac{q^{n}}{(n)_{q} !(1-q)^{n}} z^{n} .
\end{aligned}
$$

This implies the following multiplicative presentation for the universal $R$-matrix $R_{1}$ :

$$
\begin{aligned}
R_{1} & =\left((e \otimes f)\left(q^{\frac{1}{2}}-q^{-\frac{1}{2}}\right)^{2} ; q\right)_{\infty} \\
& =\prod_{n \geqq 1}\left(1-\left(q^{-\frac{1}{2}}-q^{\frac{1}{2}}\right)^{2} e \otimes f q^{n}\right) .
\end{aligned}
$$

Let us write the twisted Yang-Baxter equation explicitly for $R_{1}$ in terms of generators of $U_{q}\left(s l_{2}\right)$.

It is well known that

$$
(u+v ; q)_{\infty}=(u ; q)_{\infty}(v ; q)_{\infty}
$$

if

$$
u v=q v u
$$


The identities (10) for the universal $R$-matrix $R_{1}$ follow from (1.4.5) with

$$
u:=\left(q^{\frac{1}{2}}-q^{-\frac{1}{2}}\right)^{2} e \otimes k \otimes f, \quad v:=\left(q^{\frac{1}{2}}-q^{-\frac{1}{2}}\right)^{2}(1 \otimes e \otimes f)
$$

for upper (10) and

$$
u:=\left(q^{\frac{1}{2}}-q^{-\frac{1}{2}}\right)^{2}\left(e \otimes k^{-1} \otimes f\right), \quad v:=\left(q^{\frac{1}{2}}-q^{-\frac{1}{2}}\right)^{2}(e \otimes f \otimes 1)
$$

for lower (10).

The proof is an easy computation.

Remark. 1.4.1. As was noted at the end of the previous section, the element $R_{1}$ satisfies the twisted Yang-Baxter relation (15). In terms of function $(z ; q)_{\infty}$ this means

$$
(F ; q)_{\infty}\left(K_{+} ; q\right)_{\infty}(E ; q)_{\infty}=(E ; q)_{\infty}\left(K_{-} ; q\right)_{\infty}(F ; q)_{\infty}
$$

where

$$
\begin{gathered}
F=\left(q^{\frac{1}{2}}-q^{-\frac{1}{2}}\right)^{2} e \otimes f \otimes 1, \quad E=\left(q^{\frac{1}{2}}-q^{-\frac{1}{2}}\right)^{2} 1 \otimes e \otimes f, \\
K_{+}=\left(q^{\frac{1}{2}}-q^{-\frac{1}{2}}\right)^{2} e \otimes k \otimes f, \quad K_{-}=\left(q^{\frac{1}{2}}-q^{-\frac{1}{2}}\right)^{2} e \otimes k^{-1} \otimes f .
\end{gathered}
$$

Notice that these elements satisfy relations similar to $U_{q}\left(s l_{2}\right)$ :

$$
\begin{array}{rlrl}
K_{+} F & =q^{-1} F K_{+}, & K_{+} E=q E K_{+}, \\
K_{-} F & =q F K_{-}, & K_{-} E=q^{-1} E K_{-}, \\
{[E, F]} & =\left(K_{+}-K_{-}\right)\left(1-q^{-1}\right), \\
K_{+} K_{-} & =K_{-} K+. &
\end{array}
$$

These relations may be regarded as determining relations for $C_{q}\left[G L_{2}^{*}\right]$. The explanation of this fact remains a bit mysterious. Notice that functions $(z ; q)_{\infty}$ also appeared in $[\mathrm{FK}]$, where they were interpreted as "quantum dilogarithms."

\section{More on Quantum $s l_{2}$ Over $\mathbb{C}\left[q, q^{-1}\right]$}

Let us clarify the relation between $U_{q}\left(s l_{2}\right)$ and $U s l_{2}$.

2.1. A representation $V$ of $s l_{2}$ is said to be an integer if $H$ acts as a diagonalizable element in $V$ and if $\operatorname{Spec}\left(\left.H\right|_{V}\right) \subset \mathbb{Z}$.

Define $\dot{U} s_{2}$ as the following completion of $U s l_{2}$ in the category of integer modules [L]. It is generated by $P_{\ell}, X, Y, \ell \in \mathbb{Z}$ with determining relations

$$
\begin{gathered}
P_{\ell} P_{m}=\delta_{\ell m} P_{\ell}, \quad P_{\ell} X=X P_{\ell+2}, \\
P_{\ell} Y=Y P_{\ell-2}, \\
X Y-Y X=\sum_{\ell \in \mathbb{Z}} \ell P_{\ell}, \quad 1=\sum_{\ell \in \mathbb{Z}} P_{\ell} .
\end{gathered}
$$

As a vectorspace $\dot{U} s l_{2}$ consists of elements $\sum_{\ell, m \in \mathbb{Z}} P_{\ell} a_{\ell}(X, Y)$, where $A_{\ell}(X, Y)$ is a polynomial over $X, Y$. 
The algebra $\dot{U}_{S l} l_{2}$ is a topological Hopf algebra with the comultiplication $\Delta$ : $\dot{U} s l_{2} \rightarrow \dot{U} s l_{2} \otimes \dot{U} s l_{2}$ acting as:

$$
\begin{gathered}
\Delta(X)=X \otimes 1+1 \otimes X, \\
\Delta(Y)=Y \otimes 1+1 \otimes Y, \\
\Delta P_{\ell}=\bigoplus_{\substack{m+n=\ell \\
n, m \in \mathbb{Z}}} P_{n} \otimes P_{m} .
\end{gathered}
$$

Define the Hopf algebra $\dot{U} s l_{2}^{(\varepsilon)}$ as the algebra which is equal to $\dot{U}_{s} l_{2} \otimes_{\mathbb{C}} \mathbb{C}\left[\varepsilon^{\frac{1}{2}}\right] / \varepsilon^{2}=$ 1. The comultiplication

$$
\begin{aligned}
\Delta P_{\ell} & =\sum_{\substack{n+m=\ell \\
n, m \in \mathbb{Z}}} P_{n} \otimes P_{m}, \\
\Delta X & =X \otimes 1+\varepsilon \otimes X, \\
\Delta Y & =Y \otimes 1+\varepsilon \otimes Y, \\
\Delta \varepsilon^{\frac{1}{2}} & =\varepsilon^{\frac{1}{2}} \otimes \varepsilon^{\frac{1}{2}}
\end{aligned}
$$

provides $\dot{U} s l_{2}^{(\varepsilon)}$ with the Hopf algebra structure.

Let $\Delta$ be the usual diagonal comultiplication:

$$
\begin{aligned}
\tilde{\Delta} X & =X \otimes 1+1 \otimes X, \\
\tilde{\Delta} Y & =Y \otimes 1+1 \otimes Y, \\
\tilde{\Delta} P & =\sum_{\substack{n+m=\ell \\
n, m \in \mathbb{Z}}} P_{n} \otimes P_{m}, \\
\tilde{\Delta} \varepsilon^{\frac{1}{2}} & =\varepsilon^{\frac{1}{2}} \otimes \varepsilon^{\frac{1}{2}},
\end{aligned}
$$

which also provides a Hopf algebra structure on $\dot{U} s l_{2} \otimes_{\mathbb{C}} \mathbb{C}\left[\varepsilon \frac{1}{2}\right]$.

Consider the following element in $\dot{U} s l_{2}^{(\varepsilon) \otimes 2}$ :

$$
F=\bigoplus_{n \in \mathbb{Z}} \varepsilon^{\frac{n}{2}} \oplus P_{n}
$$

Proposition 2.1.1. (1) The comultiplication (2.1.3) is related to the diagonal comultiplication by twisting with the element $F$ :

$$
\Delta(a)=F \cdot \tilde{\Delta}(a) \cdot F^{-1} .
$$

(2) The element $F$ has the following properties:

$$
\begin{aligned}
& (\Delta \otimes \mathrm{id})(F)=F_{13} F_{23}, \\
& (\mathrm{id} \otimes \Delta)(F)=F_{13} F_{12} .
\end{aligned}
$$

The proof is by straightforward computation.

Corollary 2.1.2. The algebra $\dot{U} s l_{2}^{(\varepsilon)}$ with the comultiplication (2.1.3) is a quasitriangular Hopf algebra with

where $\sigma(a \otimes b)=b \otimes a$.

$$
R=\sigma(F) F^{-1}=\sum_{n m \in \mathbb{Z}} \varepsilon^{-\frac{m}{2}} P_{n} \otimes \varepsilon^{\frac{n}{2}} P_{m}
$$


2.2. Define the algebra $\dot{U}_{q} s l_{2}^{(\varepsilon)}$ as the $\mathbb{C}\left[q^{\frac{1}{2}}, q^{-\frac{1}{2}}\right]$-algebra generated by $e, f, \varepsilon^{\frac{1}{2}}$ and $P_{l}$ with the following determining relations:

$$
\begin{gathered}
P_{\ell} P_{m}=\delta_{\ell m} P_{\ell}, \quad 1=\sum_{\ell \in \mathbb{Z}} P_{\ell}, \\
P_{\ell} e=e P_{\ell+2}, \quad P_{\ell} f=f P_{\ell-2}, \\
e f-f e=\varepsilon \sum_{\ell \in \mathbb{Z}}(\ell)_{q^{2}} q^{-l}(q+1) P_{\ell}, \\
\varepsilon^{2}=1 .
\end{gathered}
$$

As a vectorspace $\dot{U}_{q} s l_{2}^{(\varepsilon)}$ consists of sums $\sum_{f \in \mathbb{Z}} P_{f} a_{f}\left(e, f, \varepsilon^{\frac{1}{2}}\right)$, where $a_{f}$ are polynomials over $\mathbb{C}\left[q^{\frac{1}{2}}, q^{-\frac{1}{2}}\right]$.

The following comultiplication provides $\dot{U}_{q} s l_{2}^{(\varepsilon)}$ with a Hopf algebra structure:

$$
\begin{aligned}
\Delta P_{l} & =\bigoplus_{n+m=\ell} P_{n} \otimes P_{m}, \\
\Delta e & =\sum_{\ell \in \mathbb{Z}} e \otimes q^{l / 2} \varepsilon P_{l}+1 \otimes e, \\
\Delta f & =f \otimes 1+\sum_{l \in \mathbb{Z}} q^{-\ell / 2} \varepsilon P_{\ell} \otimes f, \\
\Delta \varepsilon^{\frac{1}{2}} & =\varepsilon^{\frac{1}{2}} \otimes \varepsilon^{\frac{1}{2}} .
\end{aligned}
$$

Theorem 2.2.1. The algebra $\dot{U}_{q} s l_{2}^{(\varepsilon)}$ is quasitriangular with

$$
R=\left(\sum_{m, n \in \mathbb{Z}} q^{\frac{n m}{4}} \varepsilon^{-\frac{n}{2}} P_{m} \otimes \varepsilon^{\frac{m}{2}} P_{n}\right) \cdot \sum_{k \geqq 0} \frac{(q-1)^{k}}{(k)_{q} !} q^{\frac{k(k-1)}{2}} e^{k} \otimes f^{k}
$$

Notice that element $\varepsilon^{\frac{1}{2}}-1$ generates a Hopf ideal. Let $\dot{U}_{q}\left(s l_{2}\right)$ be the corresponding quotient algebra [L]

$$
\dot{U}_{q}\left(s l_{2}\right)=\dot{U}_{q}^{(\varepsilon)}\left(s l_{2}\right) /\left\langle\varepsilon^{\frac{1}{2}}-1\right\rangle
$$

Remark. 2.2.2. It is clear that quasitriangular Hopf algebra $\left(\dot{U}_{q}^{(\varepsilon)}\left(s l_{2}\right), \Delta, R\right)$ is a deformation of Hopf algebra $\left(U^{(\varepsilon)}\left(s l_{2}\right), \Delta, R\right)$ described in the previous section:

$$
\dot{U}_{q}^{(\varepsilon)}\left(s l_{2}\right)[[q-1]] /(q-1) \dot{U}_{q}^{(\varepsilon)}\left(s l_{2}\right)[[q-1]] \simeq U^{(\varepsilon)}\left(s l_{2}\right)
$$

Proposition 2.2.3. (1) There is an isomorphism of algebras $\phi: \dot{U}_{q}\left(s l_{2}\right)^{(\varepsilon)} \stackrel{\sim}{\rightarrow}$ $\dot{U}_{q}\left(s l_{2}\right) \otimes_{\mathbb{C}} \mathbb{C}\left[\varepsilon^{\frac{1}{2}}\right]$

$$
\phi\left(P_{\ell}\right)=P_{\ell} \otimes 1, \quad \phi(e)=e \otimes \varepsilon, \quad \phi(f)=f \otimes 1
$$

(2) The map $\tilde{\Delta}: \dot{U}_{q}\left(s l_{2}\right)^{(\varepsilon)} \rightarrow \dot{U}_{q}\left(s l_{2}\right)^{(\varepsilon)}{ }^{\otimes 2}$, 


$$
\begin{aligned}
\tilde{\Delta} P_{\ell} & =\bigoplus_{n+m=\ell} P_{n} \otimes P_{m}, \\
\tilde{\Delta} e & =\sum_{\ell \in \mathbb{Z}} e \otimes q^{\ell / 2} P_{\ell}+1 \otimes e, \\
\tilde{\Delta} f & =f \otimes 1+\sum_{l \in \mathbb{Z}} q \otimes q^{\ell / 2} P_{\ell} \otimes f, \\
\tilde{\Delta} \varepsilon^{\frac{1}{2}} & =\varepsilon^{\frac{1}{2}} \otimes \varepsilon^{\frac{1}{2}}
\end{aligned}
$$

determines a Hopf algebra structure on $\dot{U}_{q}\left(s l_{2}\right)^{(\varepsilon)}$ and it is related to the comultiplication (2.2.2) by the twist

$$
\tilde{\Delta}(\phi(a))=F^{-1}(\phi \otimes \phi)(\Delta(a)) F,
$$

where $F$ is the element (2.1.5)

(3) The Hopf algebra $\left(\dot{U}_{q}\left(s l_{2}\right)\left[\varepsilon^{\frac{1}{2}}\right], \tilde{\Delta}\right)$ is quasitriangular with

$$
\tilde{R}=\left(\sum_{n, m \in \mathbb{Z}} q^{\frac{n m}{4}} \cdot P_{m} \otimes P_{n}\right) \sum_{k \geqq 0} \frac{(q-1)^{k}}{(k)_{q} !} \cdot q^{\frac{k(k-1)}{2}} e^{k} \otimes f^{k} .
$$

Proof. Statements (1) and (2) are an easy straightforward exercise. The statement (3d) follows from general facts about twistings of quasitriangular Hopf algebras [Dr]. For the twisted $R$-matrix we have:

$$
R=F_{21} \tilde{R} F^{-1} \text {. }
$$

Formula (2.2.8) follows from relations:

$$
\begin{aligned}
& F_{21} P_{n} \otimes P_{m} F^{-1}=\varepsilon^{-\frac{m}{2}} P_{n} \otimes P_{m} \varepsilon^{\frac{n}{2}}, \\
& F_{21} e^{k} \otimes f^{k} F^{-1}=(\varepsilon e)^{k} \otimes f^{k} .
\end{aligned}
$$

Remark. 2.2.4. The quasitriangular Hopf algebra $\dot{U}_{q} s l_{2}$ is a deformation of $\dot{U}_{s} l_{2}$ : $\dot{U}_{q}\left(s l_{2}\right)[[q-1]] /(q-1) \dot{U}_{q}\left(s l_{2}\right)[[q-1]] \simeq \dot{U}_{s} l_{2}$.

\section{Quantum $s l_{2}$ at Roots of 1}

3.1. Let $\varepsilon$ be a primitive root of 1 of degree $\ell$ and let $\ell$ be odd. Let $q=e^{h} \varepsilon$ and consider

$$
U_{\varepsilon}\left(s l_{2}\right)=U_{q}\left(s l_{2}\right)[[h]] / h U_{q}\left(s l_{2}\right)[[h]] .
$$

If $A$ is an algebra we denote by $Z(A)$ its center.

Proposition 3.1.1. (1) Elements $e^{\prime}, k^{\prime}, f^{\prime}$ belong to the center of $U_{\varepsilon}\left(s l_{2}\right)$.

(2) The center of $U_{\varepsilon}\left(s l_{2}\right)$ is generated by $e^{\prime}, k^{\prime}, f^{\prime}$ and by

$$
c=e f+\frac{k+k^{-1} \varepsilon}{(\varepsilon-1)^{2}}
$$

freely modulo relation 


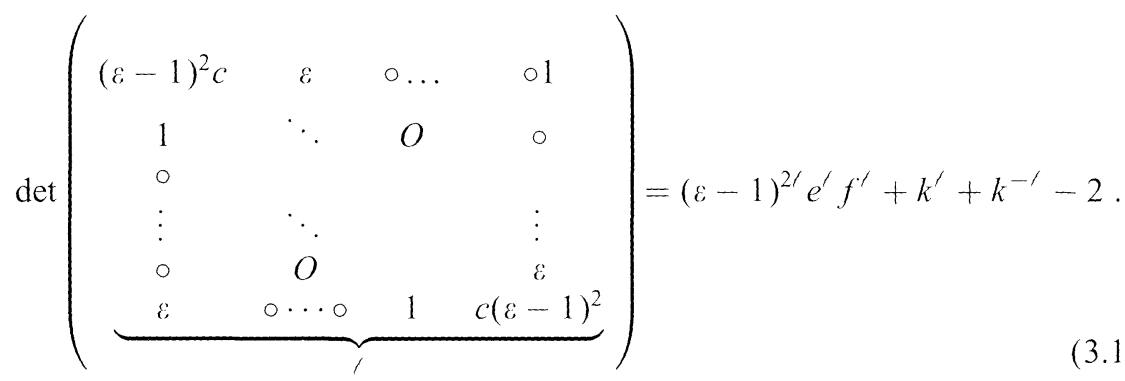

(3) The algebra $U_{i}\left(s l_{2}\right)$ is finite-dimensional over $Z\left(U_{i}\left(s l_{2}\right)\right)$.

See $[D K]$ for details.

One can introduce the Poisson structure on the center of $U_{\varepsilon}\left(s l_{2}\right)$ according to the following general construction.

Let $A_{h}$ be an algebra deformation of associative algebra $A$. We assume that $A_{h}=A[[h]]$. We denote the multiplication in $A_{h}$ by $m_{h}: A[[h]]^{\otimes 2} \rightarrow A[[h]]$ and we have $m_{h}=m \bmod h$, where $m$ is the multiplication in $A$.

Proposition 3.1.2. Let $a, b \in A[[h]]$ and either $a \bmod h$ or $b \bmod h$ belongs to the center of $A$. Then the element

$$
\{a \bmod h, b \bmod h\}=\frac{1}{h}\left(m_{h}(a, b)-m_{a}(b, a)\right) \bmod h
$$

is defined and $\{\cdot, \cdot\}$ determines a Poisson structure on the algebra $Z(A)$, and $\{\cdot, \cdot\}$ determines a Poisson action of the Poisson algebra $(Z(A),\{\cdot, \cdot\})$ by derivations of $A$.

Proof is straightforward (see for example $[D P]$ ). Notice that $\{\cdot, \cdot\}$ may be identically zero.

In our case $A$ is $U_{i:}\left(s l_{2}\right)$ and $A_{h}$ is $U_{i c h}\left(s l_{2}\right)$ and the Poisson structure $\{\cdot, \cdot\}$ can be computed explicitly between generators of $Z\left(U_{i:}\left(s l_{2}\right)\right)$. The answer is:

$$
\begin{aligned}
\left\{k^{\prime}, e^{\prime}\right\} & =\ell^{2} k^{\prime} e^{\prime}, \quad\left\{k^{\prime}, f^{\prime}\right\}=-\ell^{2} k^{\prime} f^{\prime}, \\
\left\{e^{\prime}, f^{\prime}\right\} & =\left(\frac{1}{\varepsilon-1}\right)^{2 /+1} \cdot f^{2} \cdot\left(k^{\prime}-k^{-\prime}\right), \\
\{c, a\} & =0
\end{aligned}
$$

for each $a \in Z\left(U_{2}\left(s l_{2}\right)\right)$.

The Poisson action of $Z\left(U_{\varepsilon}\left(s l_{2}\right)\right)$ on $U_{\varepsilon}\left(s l_{2}\right)$ can also be computed explicitly:

$$
\begin{aligned}
& \left\{k^{\prime}, e\right\}=l e k^{\prime}, \quad\left\{k^{\prime}, f\right\}=-\ell f k^{\prime}, \\
& \left\{k, e^{\prime}\right\}=\ell k \ell^{\prime}, \quad\left\{e^{\prime}, f\right\}=\frac{\ell}{\varepsilon-1} e^{\prime-1} \cdot \frac{k-k^{-1} \varepsilon}{\varepsilon-1}, \\
& \left\{k, f^{\prime}\right\}=-l k f^{\prime}, \quad\left\{e, f^{\prime}\right\}=\frac{\ell}{\varepsilon-1} \cdot \frac{k-k^{-1} \varepsilon}{\varepsilon-1} \cdot f^{\prime-1}, \\
& \{c, e\}=\{c, k\}=\{c, f\}=0 .
\end{aligned}
$$


All these formulas can be easily derived from relations in $U_{q}\left(s l_{2}\right)$ and from the identity

$$
\begin{aligned}
e^{\prime} f^{\prime} & =\sum_{m=0}^{\prime} \frac{(n)_{q} !}{(n-\ell+m)_{q} !} \frac{k q^{m-1+1}-k^{-1} q^{-m+1-n}}{q-1} \ldots \\
& \ldots \frac{k-k^{-1} q^{\prime-m-n}}{q-1} \cdot f^{n-1+m} \cdot e^{m},
\end{aligned}
$$

which can be found in $[\mathrm{K}]$.

Denote by $Z_{0}\left(U_{\varepsilon}\left(s l_{2}\right)\right)$ the central subalgebra in $U_{\varepsilon}\left(s l_{2}\right)$ generated by $e^{\prime}, f^{\prime}, k^{ \pm \prime}$.

Remark. 3.1.3. The algebra $Z\left(U_{\varepsilon}\left(s l_{2}\right)\right)$ is finite-dimensional over $Z_{0}\left(U_{\varepsilon}\left(s l_{2}\right)\right)$.

Proposition 3.1.4. The subalgebra $Z_{0}\left(U_{\varepsilon}\left(s l_{2}\right)\right)$ is a Hopf subalgebra with the comultiplication

$$
\begin{aligned}
& \Delta k^{\prime}=k^{\prime} \otimes k^{\prime}, \\
& \Delta e^{\prime}=e^{\prime} \otimes k^{\prime}+1 \otimes e^{\prime}, \\
& \Delta f^{\prime}=f^{\prime} \otimes 1+k^{-\prime} \otimes f^{\prime} .
\end{aligned}
$$

The proof is an elementary corollary of the identities

$$
\begin{aligned}
\Delta f^{n} & =\sum_{s=0}^{n}\left(\begin{array}{l}
n \\
s
\end{array}\right)_{q} f^{s} k^{-n+s} \otimes f^{n-s}, \\
\Delta e^{n} & =\sum_{s=0}^{n}\left(\begin{array}{l}
n \\
s
\end{array}\right)_{q} e^{s} \otimes e^{n-s} k^{s}, \\
\Delta k^{n} & =k^{n} \otimes k^{n} .
\end{aligned}
$$

The following is a general fact about Hopf algebra deformations. Let $A$ be a Hopf algebra with multiplication $m$ and comultiplication $\Delta$. Let $A_{h}$ be a Hopf algebra deformation of $A$ such that $A_{h}=A[[h]]$ as a vectorspace, $\Delta_{h}=\Delta \bmod h$ and $m_{h}=m$ $\bmod h$.

Proposition 3.1.5. Let $X(A) \subset Z(A)$ be a central subalgebra which is a Hopf subalgebra and let $\{\cdot, \cdot\}$ be the Poisson structure (3.1.4) on $X(A)$. Then $X(A)$ is a Hopf-Poisson algebra in the sense of $[\mathrm{Dr}]$ :

$$
\Delta(\{a, b,\})=\{\Delta(a), \Delta(b)\},
$$

where $\{a \otimes b, c \otimes d\}=\{a, c\} \otimes b d+a c \otimes\{b, d\}$.

Proof. Let us compute $\Delta(\{a, b\})$ :

$$
\begin{aligned}
\Delta(\{a, b\}) & =\frac{1}{h} \Delta\left(m_{h}(a, b)-m_{h}(b, a)\right) \bmod h \\
& =\frac{1}{h}\left(m_{h}\left(a^{(1)}, b^{(1)}\right) m_{h}\left(a^{(2)}, b^{(2)}\right)-m_{h}\left(b^{(1)}, a^{(1)}\right) m_{h}\left(b^{(2)}, a^{(2)}\right)\right) \bmod h \\
& =\left\{a^{(1)}, b^{(1)}\right\} m\left(a^{(2)}, b^{(2)}\right)+m\left(a^{(1)}, b^{(1)}\right)\left\{a^{(2)}, b^{(2)}\right\} \\
& =\{\Delta(a), \Delta(b)\} .
\end{aligned}
$$

This proves the proposition. 
Corollary 3.1.6. The central subalgebra $Z_{0}\left(U_{\varepsilon}\left(s l_{2}\right)\right)$ is a Hopf-Poisson algebra with the comultiplication (3.1.8) and with Poisson structure (3.1.5).

In the case of quantum $s l_{2}$ it is also an easy explicit computation which proves that (3.1.8) is compatible with Poisson brackets (3.1.5). It is also known that the coalgebra structure on $U_{q}\left(s l_{2}\right), \quad q \in \mathbb{C}^{*}$ does not depend on $q$ [FRT].

3.2. Let $\mathfrak{U}$ be a Lie algebra with Lie bracket $[\cdot, \cdot]$. Denote by $\left.H(x, y)_{[\cdot,} \cdot\right]$ its Campbell-Hausdorff series; so that

$$
\begin{aligned}
\exp (x) \cdot \exp (y) & \left.=\exp \left(H(x, y)_{[} \cdot, \cdot\right]\right) \\
H(x, y)_{[\cdot, \cdot]} & =x+y+\frac{1}{2}[x, y]+\cdots .
\end{aligned}
$$

Here we assumed $\mathfrak{A} \hookrightarrow U \mathfrak{A}$ and the multiplication in the left side is taken in $U \mathfrak{A}$. If $\mathfrak{U}$ is infinite-dimensional $U \mathfrak{U}$ should be properly defined as a topological algebra.

Another important fact is the identity

$$
\exp (x) \cdot y \cdot \exp (-x)=\sum_{n=0}^{\infty} \frac{1}{n !} \underbrace{[x[\ldots[x, y] \ldots]}_{n}
$$

which holds in $U \mathscr{A}$ (or in appropriate topological algebra).

Suppose $(A,\{\cdot, \cdot\})$ is a Poisson algebra and $A_{h}$ is an associative algebra which is a formal deformation of $(A,\{\cdot, \cdot\})$. Assume the identification of vectorspaces $A_{h}=A[[h]]$. Denote the multiplication in $A_{h}$ as $m_{h}: A_{h}^{\otimes 2} \rightarrow A_{h}$. We have

$$
m_{h}(a, b)=a b+\frac{h}{2}\{a, b\}+o\left(h^{2}\right)
$$

where $(a, b) \hookrightarrow a b$ is the commutative multiplication in $A[[h]]$.

Introduce the following Lie algebra structure on $A_{h}\left[h^{-1}\right]$ :

$$
[x, y]=\frac{1}{h}\left(m_{h}(x, y)-m_{h}(y, x)\right)
$$

For $x \in A[[h]]$ define $x_{0}=x \bmod h, x_{0} \in A$. Clearly

$$
[x, y]_{0}=\left\{x_{0}, y_{0}\right\}
$$

where $\left.x, y \in A[[h]] \hookrightarrow A\left[h^{-1}, h\right]\right]$.

In order to define Campbell-Hausdorff series for the Lie algebra $\left(A_{h},[\cdot, \cdot]\right)$ we have to consider an appropriate completion of $A$ when $A$ is infinite-dimensional. For example we can assume that $A_{h}$ is a filtered algebra with filtration $\left\{A_{h}^{(n)}\right\}$ and then

$$
\left[A^{(n)}, A^{(m)}\right] \subset A^{(n+m-1)} .
$$

In this case $\hat{A}_{h}$ will be the completion of $A_{h}$ with respect to this filtration. 
Lemma 3.2.1. Let $x, y \in A[[h]] \hookrightarrow A\left[\left[h^{-1}, h\right]\right] \hookrightarrow \hat{A}\left[\left[h^{-1}, h\right]\right]$. Then the product

$$
m_{h}\left(\exp \left(\frac{x}{h}\right), \exp \left(\frac{y}{h}\right)\right)=\exp \left(\frac{H(x, y)_{[\cdot, \cdot]}}{h}\right)
$$

is defined in $\hat{A}\left[\left[h^{-1}, h\right]\right]$. Here $H(x, y)$ is the Campbell-Hausdorff series for Lie algebra $\left(\hat{A}_{h},[\cdot, \cdot]\right)$.

Note that (3.2.5) implies

$$
H(x, y)_{[\cdot, \cdot]} \bmod h=H\left(x_{0}, y_{0}\right)_{\{\cdot, \cdot\}},
$$

where $x, y \in \hat{A}[[h]]$.

Lemma 3.2.2. For $x, y \in A_{h} \hookrightarrow A\left[\left[h^{-1}, h\right]\right]$, the product

$$
m_{h}\left(m_{h}\left(\exp \left(\frac{x}{h}\right), y\right), e^{-\frac{x}{h}}\right)=: \exp (x) * y
$$

is defined over $\hat{A}\left[\left[h^{-1}, h\right]\right]$ and $\exp (x) * y \in \hat{A}[[h]] \hookrightarrow \hat{A}\left[\left[h^{-1}, h\right]\right]$.

This lemma follows immediately from (3.2.2) and from the fact that $x y-y x=0$ $\bmod h$.

Notice that

$$
\exp (x) * y \bmod h=\exp \left(x_{0}\right) \circ y_{0}
$$

where

$$
\exp \left(x_{0}\right) \circ y_{0}=\sum_{n \geqq 0} \frac{1}{n !} \underbrace{\left\{x _ { 0 } \left\{\ldots \left\{x_{0},\right.\right.\right.}_{n} y_{0}\} \ldots\} .
$$

3.3. Let $S L_{2}^{*}$ be a Lie group dual to $S L_{2}$ in the sense of dual Lie-Poisson groups [Dr]. This group may be regarded as a group of pairs of triangular matrices:

$$
\left(\left(\begin{array}{ll}
1 & e \\
0 & k
\end{array}\right),\left(\begin{array}{cc}
1 & 0 \\
f & k^{-1}
\end{array}\right)\right)
$$

with pairwise multiplication $(x, y)\left(x^{\prime}, y^{\prime}\right)=\left(x x^{\prime}, y y^{\prime}\right)$.

This group is a Lie-Poisson group [Dr] which means, in particular, that the algebra of algebraic function $C\left[S L_{2}^{*}\right]$ is a Hopf-Poisson algebra with the comultiplication induced by a group multiplication in $S L_{2}^{*}$ and with the following Poisson brackets between coordinate functions:

$$
\{e, f\}=k-k^{-1}, \quad\{k, e\}=k e, \quad\{k, f\}=-k f .
$$

Remark. 3.3.1. We have an isomorphism of coalgebras (see for example [FRT]):

$$
U_{q}\left(s l_{2}\right) \otimes_{\mathbb{C}\left[q, q^{-1}\right]} \mathbb{C}(q) \simeq C\left[S L_{2}^{*}\right](q) .
$$

Proposition 3.3.2. There is an isomorphism of Hopf-Poisson algebras:

$$
\begin{gathered}
\phi: Z_{0}\left(U_{\varepsilon}\left(s l_{2}\right)\right) \stackrel{\sim}{\longrightarrow} C\left[S L_{2}^{*}\right], \\
\phi\left(e^{\ell}\right)=(\varepsilon-1)^{-\ell-1} e, \quad \phi\left(k^{\ell}\right)=k, \quad \phi\left(f^{\ell}\right)=(\varepsilon-1)^{-\ell} f, \\
\phi(\{a, b\})=\ell^{2}\{\phi(a), \phi(b)\} .
\end{gathered}
$$


The proof is clear.

Let $C\left[\left[S L_{2}^{*}\right]\right]$ be the algebra of jets of functions on $S L_{2}^{*}$ in the neighbourhood of 1 . As a vectorspace it consists of the formal power series over $k-1, e, f$.

Theorem 3.3.3. The algebra $C\left[\left[S L_{2}^{*}\right]\right]$ is braided with the automorphism $R$ of the form:

$$
R(a)=\exp \left(r_{0}\right) \circ \exp \left(r_{1}\right) \circ a
$$

Here

$$
\begin{gathered}
r_{0}=\frac{1}{4} z \otimes z, \\
r_{1}=L i_{2}(e \otimes f), \quad L i_{2}(x)=-\int_{0}^{x} \frac{\log (1-y)}{y} d y,
\end{gathered}
$$

and we assumed $k=\exp \left(\frac{z}{2}\right)$.

Proof. Consider the algebra $C_{h}\left[\left[S L_{2}^{*}\right]\right]$ over $\mathbb{C}[[h]]$ generated by $\bar{e}, \bar{f}, z$ (we will assume that it is completed by formal power series with respect to $\bar{e}, \bar{f}$ and $z$ ) with determining relations:

$$
\begin{aligned}
& \bar{e} \bar{f}-\bar{f} \bar{e}=\left(e^{\frac{h}{2}}-e^{-\frac{h}{2}}\right)\left(e^{\frac{z}{2}}-e^{-\frac{z}{2}}\right), \\
& {[z, \bar{e}]=2 h e, \quad[z, \bar{f}]=-2 h \bar{f},}
\end{aligned}
$$

It is a Hopf algebra with the comultiplication

$$
\begin{aligned}
\Delta z & =z \otimes 1+1 \otimes z, \\
\Delta \bar{e} & =\bar{e} \otimes e^{\frac{z}{2}}+1 \otimes \bar{e}, \\
\Delta \bar{f} & =\bar{f} \otimes 1+e^{-\frac{z}{2}} \otimes \bar{f} .
\end{aligned}
$$

Clearly this algebra is a Hopf algebra deformation of Hopf-Poisson algebra $C\left[\left[S_{2}^{*}\right]\right]$, and we have the isomorphism of vector spaces $C_{h}\left[\left[S L_{2}\right]\right] \simeq \mathbb{C}[[\mathbb{C}[[k-1]] \otimes$ $\mathbb{C}[[f]] \otimes \mathbb{C}[[h]]$.

The map $\phi: U_{q}\left(s l_{2}\right) \rightarrow C_{h}\left[\left[S L_{2}^{*}\right]\right]\left[h^{-1}\right]$

$$
\phi(e)=\frac{\bar{e}}{\left(e^{\frac{h}{2}}-e^{-\frac{h}{2}}\right)}, \quad \phi(f)=\frac{\bar{f}}{\left(e^{\frac{h}{2}}-e^{-\frac{h}{2}}\right)}, \quad \phi(k)=e^{\frac{z}{2}}
$$

is a homomorphism of algebras. Here we assumed that $q=e^{h}$ and considered $U_{q}\left(s l_{2}\right)$ over $\mathbb{C}[[h]]$.

Consider the image of $R_{1}$ under the extension of $\phi \otimes \phi$ to an appropriate completion of $U_{q}\left(s l_{2}\right)^{\otimes 2}$ :

$$
(\phi \otimes \phi)\left(R_{1}\right)=\left(\bar{e} \otimes \bar{f} ; e^{h}\right)_{\infty} .
$$

Here $\left(z ; e^{h}\right)_{\infty}$ is the asymptotics of the function $(z ; q)_{\infty}$ at $q \rightarrow 1$ (see the lemma from Sect. 3.4)

$$
\left(\bar{e} \otimes \bar{f} ; e^{h}\right)=\exp \left(\frac{L i_{2}(\bar{e} \otimes \bar{f})}{h}\right)(1-\bar{e} \otimes \bar{f})^{\frac{1}{2}} \cdot(1+O(h)) .
$$

This asymptotics in the element of $\mathbb{C}_{h}\left[\left[S L_{2}^{*}\right]\right]^{\hat{\otimes} 2}\left[\left[h^{-1}\right]\right]$, where the tensor product completed by power series over $\bar{e} \otimes 1,1 \otimes \bar{e}, \ldots$.

According to the previous section the element

$$
R^{(1)}(a)=(\phi \otimes \phi)\left(R_{1}\right) \cdot a \cdot(\phi \otimes \phi)\left(R_{1}\right)^{-1},
$$


where $a \in C_{h}\left[\left[S L_{2}^{*}\right]\right]^{\otimes 2}$ exists in $C_{h}\left[\left[S L_{2}^{*}\right]\right]^{\otimes 2}$ and determines the algebra automorphism of $C_{h}\left[\left[S L_{2}^{*}\right]\right]^{\hat{\otimes} 2}$.

We will denote by $R^{(0)}$ the image of the automorphism (1.2.6)-(1.2.8) under $\phi \otimes \phi:$

$$
\begin{array}{ll}
R^{(0)}(\bar{e} \otimes 1)=\bar{e} \otimes e^{\frac{z}{2}}, & R^{(0)}(\bar{f} \otimes 1)=\bar{f} \otimes e^{-\frac{z}{2}}, \\
R^{(0)}(1 \otimes \bar{e})=e^{\frac{z}{2}} \otimes \bar{e}, & R^{(0)}(1 \otimes \bar{f})=e^{-\frac{z}{2}} \otimes \bar{f}, \\
R^{(0)}(z \otimes 1)=z \otimes 1 \sigma, & R^{(0)}(1 \otimes z)=1 \otimes z,
\end{array}
$$

Define

$$
\bar{R}(a)=R^{(0)} \circ R^{(1)}(a) .
$$

From the definition of $R$ and from the braiding of $U_{q}\left(s l_{2}\right)$ we deduce that $C_{h}\left[\left[S L_{2}^{*}\right]\right]$ is braided with $\bar{R}$ defined in (3.3.17).

Moreover, we can represent $R^{(0)}$ as follows:

$$
R^{(0)}(a)=\exp \left(\frac{z \otimes z}{4 h}\right) \cdot a \cdot \exp \left(-\frac{z \otimes z}{4 h}\right) .
$$

As was explained in the previous section this product exists in $C_{h}\left[\left[S L_{2}^{*}\right]\right]^{\otimes 2} \hookrightarrow$ $C_{h}\left[\left[S L_{2}^{*}\right]\right]^{\hat{\otimes} 2}\left[\left[h^{-1}\right]\right]$.

Since the Hopf algebra $C_{h}\left[\left[S L_{2}^{*}\right]\right]$ is a deformation of Hopf-Poisson algebra $C_{h}\left[\left[S L_{2}^{*}\right]\right]$ the latter is braided with

$$
R(a \bmod h)=\bar{R}(a) \bmod h
$$

and since (3.3.14), (3.3.15) and (3.3.18)

$$
R(a)=\exp \left(r_{0}\right) \circ \exp \left(r_{1}\right) \circ a,
$$

where $\exp (a) \circ b$ is defined in (3.2.11).

Note that in the proof of Theorem 3.3.3 we have also proven that $C_{h}\left[\left[S L_{2}^{*}\right]\right]$ is a braided Hopf algebra.

Remark. 3.3.4. The analogue of Theorem 3.3.3 for an arbitrary simple Lie algebra (5) with the standard Lie bialgebra structure [Dr] has been given in [R], where linear terms of $r_{0}$ and $r_{1}$ have been described.

3.4. Let $(z ; q)_{\infty}$ be the function defined in (1.4.1) for $|q|<1$.

Lemma 3.4.1. The function $(z ; q)_{\infty}$ has the following asymptotics when $q \rightarrow \varepsilon$, $\varepsilon^{\ell}=1, \ell$-odd:

$$
\begin{aligned}
(z ; q)_{\infty} & \rightarrow \exp \left(-\frac{1}{\ell^{2} h} \int_{0}^{z^{\ell}} \frac{\log (1-t)}{t} d t\right) \\
& \cdot\left(1-z^{\ell}\right)^{\frac{1}{2}} \prod_{m=0}^{\ell}\left(1-\varepsilon^{m} z\right)^{-\frac{m}{\ell}}(1+O(h))
\end{aligned}
$$

where $q=\varepsilon e^{h}, h \rightarrow 0$. 
Proof. The function $(z ; q)_{\infty}$ satisfies the following difference equation:

$$
(z q ; q)_{\infty}=\frac{1}{1-z q}(z ; q)_{\infty},
$$

and it is uniquely determined by this property and by the condition $(0 ; q)_{\infty}=1$. Iterating this equation we have

$$
\left(z q^{\ell} ; q\right)_{\infty}=\frac{1}{(1-z q) \ldots\left(1-z q^{\prime}\right)}(z ; q)_{\infty} .
$$

The function (3.4.1) presents the asymptotics of the solution to Eq. (3.4.3) normalized by $(0 ; q)_{\infty}=1$.

3.5. Let $\varepsilon$ be a root of 1 of odd degree $\ell$.

Definition 3.5.1. The algebra $\overline{U_{\varepsilon}\left(s l_{2}\right)}$ is a complex algebra generated by elements $\bar{e}, \bar{f}, \omega, z$ (complicated by formal power series over $\bar{e}, \bar{f}, z$ ) with the following determining relations:

$$
\begin{aligned}
& \omega \bar{e}=\varepsilon \bar{e} \omega, \quad \omega \bar{f}=\varepsilon^{-1} \bar{f} \omega, \\
& \omega^{\ell}=1, \quad \bar{e} \bar{f}-\bar{f} \bar{e}=\frac{\omega e^{\frac{z}{2}}-\omega^{-1} e^{-\frac{z}{2}}}{\varepsilon-1},
\end{aligned}
$$

and $z \in$ center of $\overline{U_{\varepsilon}\left(s l_{2}\right)}$.

Clearly $\overline{U_{\varepsilon}\left(s l_{2}\right)}$ is a Hopf algebra with the comultiplication

$$
\begin{aligned}
\Delta \bar{e} & =\bar{e} \otimes \omega e^{\frac{z}{2}}+1 \otimes \bar{e}, \\
\Delta \bar{f} & =\bar{f} \otimes 1+\omega^{-1} e^{-\frac{z}{2}} \otimes \bar{f}, \\
\Delta z & =z \otimes 1+1 \otimes z,
\end{aligned}
$$

and the map $\phi: U_{\varepsilon}\left(s l_{2}\right) \rightarrow \overline{U_{\varepsilon}\left(s l_{2}\right)}$,

$$
\phi(k)=\omega e^{\frac{z}{2}}, \quad \phi(e)=\bar{e}, \quad \phi(f)=\bar{f}
$$

determines a homomorphism of Hopf algebras.

The algebra $\overline{U_{\varepsilon}\left(s l_{2}\right)}$ has all properties absolutely similar to $U_{\varepsilon}\left(s l_{2}\right)$ :

- The center of $\overline{U_{\varepsilon}\left(s l_{2}\right)}$ is generated by $\bar{e}^{\ell}, \bar{f}^{\ell}, z$ and by

$$
c=e f+\frac{\omega e^{\frac{z}{2}}+\varepsilon \omega^{-1} e^{-\frac{z}{2}}}{(\varepsilon-1)^{2}} .
$$

- $Z\left(\overline{U_{\varepsilon}\left(s l_{2}\right)}\right)$ is generated by $\bar{e}^{\ell}, \bar{f}^{\ell}, z, c$ freely modulo the relation (3.1.3) where we have to replace $k \rightarrow e^{\frac{z}{2}} \omega$.

- $\overline{U_{\varepsilon}\left(s l_{2}\right)}$ is finite-dimensional over $Z\left(\overline{U_{\varepsilon}\left(s l_{2}\right)}\right)$.

- The central subalgebra $Z_{0}\left(\overline{U_{\varepsilon}\left(s l_{2}\right)}\right)$ is a Hopf subalgebra in $\overline{U_{\varepsilon}\left(s l_{2}\right)}$ and Poisson brackets (3.1.5), (3.1.6) determine the structure of Hopf-Poisson algebra on $Z_{0}\left(\overline{U_{\varepsilon}\left(s l_{2}\right)}\right)$ together with its Poisson action on $\overline{U_{\varepsilon}\left(s l_{2}\right)}$.

- There is an isomorphism of Hopf-Poisson algebras $Z_{0}\left(\overline{U_{\varepsilon}\left(s l_{2}\right)}\right) \simeq C\left[\left[S L_{2}^{*}\right]\right]$. 
Let $\varepsilon^{\frac{1}{4}}$ be the $4 \ell^{\text {th }}$-root of 1 , such that $\varepsilon^{\frac{\ell}{4}}:=\left(\varepsilon^{\frac{1}{4}}\right)^{\ell}=i$ and consider $\overline{U_{\varepsilon}\left(s l_{2}\right)}$ over $\mathbb{C}\left[\varepsilon^{\frac{1}{4}}, \varepsilon^{-\frac{1}{4}}\right]$. Then we have:

Theorem 3.5.2. (1) The algebra $\overline{U_{\varepsilon}\left(s l_{2}\right)}$ is braided Hopf algebra with

$$
R(a)=R^{(0)}\left(R^{(1)} a R^{(1)^{-1}}\right)
$$

where

$$
\begin{gathered}
R^{(0)}(a)=\exp \left(r_{0}\right) \circ \exp \left(r_{1}\right) \circ a, \\
r_{0}=\frac{1}{4} z \otimes z, \quad r_{1}=\frac{1}{\ell^{2}} L i_{2}\left(\bar{e}^{\ell} \otimes \bar{f}^{\prime}\right),
\end{gathered}
$$

and

$$
R^{(1)}=\left(\sum_{s, t=0}^{4 t-1} \varepsilon^{\frac{s t}{4}} \omega^{s} \otimes \omega^{t}\right) \prod_{m=0}^{\ell}\left(1-\varepsilon^{m} \bar{e} \otimes \bar{f}\right)^{-\frac{m}{l}} .
$$

(2) The element $R^{(1)}$ satisfies relations (12),(13).

Proof. First, it is easy to check that the automorphism

$$
R^{(0)}=\left(\left(\sum_{s, t=0}^{4 \ell-1} \varepsilon^{\frac{s t}{4}} \omega^{s} \otimes \omega^{t}\right) a\left(\sum_{s, t=0}^{4 t-1} \varepsilon^{\frac{s t}{4}} \omega^{s} \otimes \omega^{t}\right)^{-1}\right)
$$

acts as (1.3.1)-(1.3.3) (assuming there $k^{ \pm 1}=\omega^{ \pm 1} e^{ \pm \frac{z}{2}}$ ).

Then notice that when $q=e^{h} \varepsilon, h \rightarrow 0$, the asymptotics of the element (1.2.13) is given by

$$
\exp \left(\frac{1}{h \ell^{2}} L i_{2}\left(\bar{e}^{\ell} \otimes \bar{f}^{\prime}\right)\right)\left(1-\bar{e}^{\ell} \otimes \bar{f}^{\prime}\right)^{\frac{1}{2}} \prod_{m=0}^{-1}\left(1-\varepsilon^{m} \bar{e} \otimes \bar{f}\right)^{-\frac{m}{\ell}}(1+O(h))
$$

This follows from Sect. 3.4.

The theorem now follows from Proposition 1.2.3 and from (3.2.9), (3.2.10).

3.6. Let us discuss the relation of the algebra $\overline{U_{\varepsilon}\left(s l_{2}\right)}$ described in Theorem 3.5 .2 to the finite dimensional quotient algebra

$$
U_{\varepsilon}\left(s l_{2}\right)^{\prime}=\overline{U_{\varepsilon}\left(s l_{2}\right)} /\left\langle\bar{e}^{\prime}, \bar{f}^{\prime}, z\right\rangle
$$

where $\left\langle e^{\prime}, f^{\prime}, z\right\rangle$ is the ideal generated by these elements.

It is well known that the algebra $U_{\varepsilon}\left(s l_{2}\right)^{\prime}$ is a quasitriangular finite dimensional Hopf algebra with the universal $R$-matrix

$$
\bar{R}=\left(\sum_{s, t=0}^{4 \ell-1} \varepsilon^{\frac{s t}{4}} \omega^{s} \otimes \omega^{t}\right) \sum_{n=0}^{\ell-1} \frac{\varepsilon^{\frac{k(k-1)}{2}}}{(k)_{\varepsilon} !} \cdot e^{k} \otimes f^{k} .
$$

For details see for example [RT]. The following proposition explains the relation between (3.6.2) and (3.5.8) 
Proposition 3.6.1. (1) The automorphism $R^{(0)}$ from (3.5.6) induces an identity automorphism on the quotient algebra $U_{\varepsilon}\left(s l_{2}\right)^{\prime \otimes 2}$. (2) We have the identity:

$$
\prod_{m=0}^{\ell}\left(1-\varepsilon^{m} z^{m}\right)=\sum_{n=0}^{\ell-1} \frac{\varepsilon^{\frac{k(k+1)}{2}}}{(\varepsilon-1)(k)_{\varepsilon} !} z^{n}+O\left(z^{\prime}\right) .
$$

Proof. The first statement follows immediately from the fact that point $k=1, e=0$, $f=0$ is a symplectic leaf of the Poisson structure (3.3.2) on $S L_{2}^{*}$. The identity (3.6.3) follows from the comparison of the asymptotics (3.4.1) with the representation (1.4.2) in the case when $q=\varepsilon e^{h}, h \rightarrow 0$, and $z^{\ell}=o(h)$.

Thus, the quasitriangular of the quotient algebra $U_{\varepsilon}\left(s l_{2}\right)^{\prime}$ with the universal $R$-matrix (3.6.1) is in agreement with the braiding structure of $\overline{U_{\varepsilon}\left(s l_{2}\right)}$ with the universal $R$-matrix (3.5.8).

\section{Conclusion}

We have shown that in an appropriate sense the algebra $U_{q}\left(s l_{2}\right)$ is quasitriangular even when $q$ is a root of 1: it is a braided Hopf algebra in the sense of Definition 1. This modified notion reproduces known results for appropriate quotients of $U_{q}\left(s l_{2}\right)$ [RT,R].

Notice that functions similar to those which describe the $\bar{e} \otimes \bar{f}$ dependence in (3.5.8) had already appeared in the literature in the context of the Chiral Potts model (see $[\mathrm{BB}]$ and $[\mathrm{FK}]$ ).

The generalization of these results for $U_{q}(\mathfrak{g})$ for simple Lie algebras $\mathfrak{g}$ is straightforward and will be done in a separate publication.

The following list of problems seems natural to understand now:

(i) The description of the category of modules for algebras $U_{\varepsilon}(g)$. One has to understand the category where both associative algebra and Poisson structures for $U_{\varepsilon}(\mathfrak{g})$ are taken into account.

(ii) The description of algebras $U_{\varepsilon}(\hat{\mathfrak{g}})$, where $\hat{\mathfrak{g}}$ is a Kac-Moody algebra. It is especially interesting to do this for affine Lie algebras $\hat{\mathfrak{g}}$.

First steps towards understanding question (i) have already been done in [WX] for $\varepsilon=1$. Certain results about the quantum affine algebra $U_{q}(\hat{s} l(n))$ including the description of minimal cyclic representations can be found in [DJMM].

\section{References}

[BB] Bazhanov, V.V., Baxter, V.V.: "New solvable lattice models in three dimensions." J. Stat. Phys. 69, no3/4 463-485 (1992)

[DJMM] Date, E., Jimbo, M., Miki, K., Miwa, T.: "Generalized Chiral Potts Models and Minimal Cyclic Representations of $U_{q}(\hat{s} l(n))$." Commun. Math. Phys. 137, 133-148 (1991)

[Dr] Drinfeld, V.G.: "Quantum groups." Proc. Intern. Congress of Math. (Berkeley, 1986), AMS 1987, 798-820

[Dr1] Drinfeld, V.G.: "On almost cocommutative Hopf algebras." Algebra and Analysis 1, no. 2, 30-46 (1989)

[DK] DeConcini, C., Kac, V.: "Representations of quantum groups at roots of 1." in Progress in Mathematics, Vol.92, Basel-Boston: Birkhauser, 1990 
[DP] DeConcini, C., Procesi, C.: "Quantum groups." Preprint no. 6, Scuola Normale Superiore, 1993

[FK] Faddeev, L.D., Kashaev, M. Helsinki preprint, 1993

[FRT] Faddeev, L.D., Reshetikhin, N. Yu., Takhtajan, L.A.: "Quantization of Lie groups and Lie algebras." Algebra and Analysis 1, no. 1, 178-206 (1989)

[J] Jimbo, M.: " $q$-difference analog of $U(\mathfrak{5})$ and the Yang-Baxter equation." Lett. Math. Phys. 10, 63-69 (1985)

[K] Kac, V.G.: "Infinite dimensional Lie algebras." Cambridge: Cambridge University Press, 1990

[L] Lusztig G.: Introduction to Quantum Groups. Progress in Mathematics, vol. 110, BaselBoston: Birkhauser, 1993

[R] Reshetikhin N.: "Quasitriangularity of qunatum groups and Poisson-quasitriangular HopfPoisson algebras." U.C. Berkeley preprint, 1992

[RT] Reshetikhin, N., Turaev, V.: "Invariants of 3-manifolds via link polynomials and quantum groups." Invent. Math. 103, 545-597 (1991)

[S1] Sklyanin, E.K.: "On some algebraical structures related to the Yang-Baxter equation." Funct. Anal. and its Appl. 16, no. 4, 27-34 (1982)

[S2] Sklyanin, E.K.: "On one algebra generated by quadratic relations." Usp. Mat. Nauk. 40, no. 2, 214 (1985)

[WX] Weinstein, A., Xu, W.: "Classical solutions to the quantum Yang-Baxter equation." Commun. Math. Phys. 43, 309-344 (1992)

Communicated by A. Jaffe 
